\title{
Effects of verbal pretraining and overt verbalization on discrimination learning in preschool children
}

\author{
DIANE T. LINDSLEY and JOAN H. CANTOR \\ University of Iowa, Iowa City, Iowa 52242
}

\begin{abstract}
Three groups of preschool children received relevant verbal pretraining followed by a simultaneous discrimination learning task in which two irrelevant dimensions varied within settings. In Group B, the irrelevant dimensions varied between settings in pretraining, whereas in both Group W and Group WO, the irrelevant dimensions varied within settings during pretraining. The latter groups differed only in that Group WO was required to name the relevant dimensional values overtly during discrimination learning. Discrimination performance in Group W was significantly superior to that in Group B and did not differ from that in Group WO. The results are consistent with either or both of the following assumptions: (1) Irrelevant dimensional responses were extinguished during pretraining in Group W; (2) stimulus novelty increased the occurrence of irrelevant dimensional responses during discrimination learning in Group B.
\end{abstract}

The purpose of the present study was to assess the effects of type of verbal pretraining and overt dimensional naming on the discrimination performance of preschool children. A simultaneous discrimination problem was used in which one relevant and two irrelevant dimensions varied within settings. Three groups of preschool children were taught during pretraining to name the values of the dimension that was to be relevant in the subsequent discrimination task. One way in which the groups differed was the manner in which the irrelevant dimensions varied during the pretraining task. In one group (Group B), the irrelevant dimensions varied between settings during pretraining and within settings during discrimination learning, while in the remaining two groups (Groups W and WO), the irrelevant dimensions varied within settings in both tasks. The only difference between the latter two groups was that Group WO was required to name the relevant dimensional values overtly during the discrimination task.

Verbal pretraining has repeatedly been shown to affect the subsequent discrimination performance of children (e.g., Hardesty, 1970; Miller, 1971; Spiker, 1971). It was demonstrated in these studies that pretraining with relevant dimensional labels facilitates discrimination performance, whereas pretraining with irrelevant labels interferes. At a theoretical level, these results have been explained in terms of the transfer of differential verbal or other types of cue-producing responses (CPRs)! to the values of the pretrained dimension (Spiker, 1971). The CPRs are assumed to produce distinctive stimuli, providing an added stimulus dimension that facilitates discrimination learning if it is

This report is based on an honors thesis submitted by the first author for a BS degree in psychology at the University of Iowa. The authors are indebted to Charles C. Spiker for a helpful critical reading of the manuscript. Requests for reprints should be addressed to Joan H. Cantor, Department of Psychology, University of Iowa, Iowa City, Iowa 52242. relevant and interferes if it is irrelevant.

Other types of hypothesized dimension-specific responses include observing responses (Zeaman \& House, 1963), mediated representational responses (Kendler \& Kendler, 1968), and identifying responses (Tighe \& Tighe, 1966). Although the dimension-specific responses are assumed to have somewhat different functions in the various theories, all of the theories can explain the facilitation and interference effects that have been demonstrated, given appropriate assumptions about which dimensional responses occur in a given situation. A major problem for all of the theories, however, is specification of the conditions that govern the occurrence and transfer of these responses. Some information about the conditions of transfer has been provided by the earlier studies of verbal pretraining (Spiker, 1971). Attention theorists have also been concerned with this problem (Shepp \& Turrisi, 1966), and have used intradimensional (IDS) and extradimensional (EDS) shift paradigms to help identify the relevant variables (e.g., Dickerson, Wagner, \& Campione, 1970; Kemler \& Shepp, 1971; Shepp \& Gray, 1971). Additional information comes from studies of reversal (RS), nonreversal (NRS), and optional (OS) shift learning (e.g., Kendler \& Kendler, 1961; Kendler, 1964, 1974; Spiker \& Cantor, 1973; Tighe \& Tighe, 1970).

One of the problems that has complicated the study of dimensional transfer is the intrusion of instrumental transfer. Clearly, in RS, NRS and OS paradigms, instrumental transfer affects shift performance. In EDS and IDS paradigms, the stimulus values are changed on all dimensions from the first to the second tasks. In addition, counterbalancing of the stimulus values has been employed in an attempt to cancel out potential differential generalization effects. The assumption that such counterbalancing indeed cancels the effects of differential generalization, however, remains gratuitous until it has been empirically demonstrated. It may turn 
out to be the case, for example, that the amount of generalization in a particular subgroup determines which dimension-specific responses will occur. The use of verbal pretraining provides an alternative method by which the original set of CPRs may be established. A major advantage of this method is that there is neither direct instrumental transfer nor generalization of instrumental responses from the pretraining to the discrimination task. Thus, it is not necessary to change the dimensional values after the CPRs are established, a procedure that may actually weaken the transfer of these CPRs.

One purpose of the present study was to test the generality of findings from the shift studies (Kemler \& Shepp, 1971; Shepp \& Gray, 1971) regarding the extinction of irrelevant CPRs during the initial task. Based on these results and those of earlier verbal pretraining studies, the following assumptions were made about the establishment and extinction of CPRs during pretraining in the present study. (1) For all three groups, CPRs would be established by the end of pretraining to the values of the relevant dimension of the discrimination task. (2) In Group W, CPRs would tend to occur initially to the values of the irrelevant dimensions, but they would be extinguished by the end of pretraining. (3) In Group B, since the irrelevant dimensions vary between settings, CPRs would not tend to occur to the values of these dimensions; thus, no extinction of irrelevant CPRs would occur. If these assumptions are correct, then discrimination performance should be better in Group $W$ than in Group B as a result of the extinction of irrelevant CPRs in the former group. If not extinguished, these irrelevant CPRs might successfully compete with relevant CPRs at the onset of discrimination learning. A difference between Groups W and B could be attributed not only to the extinction of irrelevant CPRs in Group W, but also to a novelty effect in Group B. Seeing the irrelevant dimensions varying within settings for the first time may increase the tendency for subjects in Group B to make irrelevant CPRs during discrimination learning. Finally, since the occurrence of relevant CPRs is guaranteed in Group WO by the requirement of overt naming, performance in this group might be expected to be superior to that in Group W, unless complete transfer of relevant CPRs occurs in the latter group.

\section{METHOD}

\section{Subjects and Design}

The subjects were 44 children (CA range of 48-69 months) from the University of Iowa Preschool Laboratories and 15 children (CA range of 53-66 months) from the Iowa City Parent's Preschool. The data for two subjects were not used, since they failed to meet the pretraining criterion. Of the remaining children, 19 were randomly assigned to each of the three verbal pretraining groups.

Each discrimination learning problem consisted of eight settings in which all dimensions varied within settings. All possible combinations of form (square or circle), size (large or small), and hue (yellow or blue) were used, and the four resulting pairs of blocks together with their lateral reversals constituted the eight settings. For approximately half the subjects, form served as the relevant dimension, with size and hue both irrelevant. For the remaining subjects, size was relevant, with form and hue both irrelevant. The factorial combination of verbal condition (B, W, WO) and relevant dimension (size, form) resulted in a 3 by 2 design. Also counterbalanced was the assignment of positive cue within each relevant dimension.

\section{Materials and Apparatus}

The stimuli were Plexiglas blocks, $4.9 \mathrm{~cm}$ tall, and halved along the frontal plane with the two halves joined at the back by a hinge. Each block could be opened by lifting the upper half, revealing a marble well that was used to bait the correct block on each trial. The large blocks were $8.9 \mathrm{~cm}$ on a side (squares) or $10.2 \mathrm{~cm}$ in diam (circles), and the small blocks were $6.4 \mathrm{~cm}$ on a side or $7.9 \mathrm{~cm}$ in diam. During the discrimination task, blocks from two virtually identical sets were interchanged at random to prevent learning on the basis of extraneous cues.

A modified Wisconsin general testing apparatus consisting of a vertical screen attached to a sliding tray was used to administer learning trials. Additional materials included a screen used to conceal the blocks during pretraining and a container into which subjects placed the marble rewards. Between trials, the experimenter baited the appropriate block and placed the appropriate pair of blocks approximately $12 \mathrm{~cm}$ apart on the tray; the blocks were presented to the subject by sliding the tray forward.

\section{Procedure}

Each subject was tested individually in a single session, first being given the appropriate verbal pretraining and then the discrimination learning task. During pretraining, the subject labeled the blocks using the values of the dimension that would be relevant in the discrimination task. For subjects in Groups W and WO, the blocks were presented in the same settings that would be used in discrimination learning, with all dimensions varying within settings. For Group B, however, the blocks were rearranged for pretraining into eight settings so that the dimensions that would be irrelevant during discrimination learning varied between settings. The subjects were brought to a pretraining criterion of one trial block in which correct names were given for all blocks in the eight settings.

Immediately after the pretraining criterion was attained the subjects was told, "Now we're going to play a marble game. This time the blocks will come out on a tray, right here. One kind of block has a marble in it every time and the other kind of block never has a marble in it. Let's see if you can learn which kind of block always has the marble .." Children in Group WO were required to tell the experimenter which block they were going to open each time, using the names learned during pretraining. A noncorrection procedure was used throughout. The settings were presented in a predetermined random order, with the restriction that each setting occur once in each eight-trial block. Discrimination learning was continued to a criterion of seven correct responses in each of two successive eight-trial blocks, with a minimum of 24 and a maximum of 40 trials.

\section{RESULTS}

The pretraining data were not analyzed, since very few naming errors were made in any of the groups. The mean proportions of correct responses for the three pretraining groups during discrimination learning are 
presented in Table 1 for each of five eight-trial blocks and for all trial blocks combined. For subjects who met criterion in less than 40 trials, the proportion of correct responses made in the final criterial block was assigned to the remaining trial blocks. It may be seen in Table 1 that performance in Group B was inferior to that in Groups $\mathrm{W}$ and WO, and that there was very little difference between the latter groups.

An initial analysis indicated that there were no significant differences in discrimination performance for form-relevant and size-relevant groups; thus, counterbalancing subgroups were combined in subsequent analyses. The verbal pretraining groups were compared in an analysis of variance with groups as a between-subjects factor and trial blocks as a within-subjects factor. The main effect of groups was significant $[F(2,54)=6.04, p<.005]$, as was the main effect of trial blocks $[\mathrm{F}(4,216)=13.91, \mathrm{p}<.0001]$. The Groups by Trial Blocks interaction was not significant $[\mathrm{F}(8,216)=.90]$. Follow-up analyses indicated that Group W performed significantly better than did Group B $[F(1,36)=8.00, p=.008]$. No significant difference was found between Groups $\mathrm{W}$ and WO $[\mathrm{F}(1,36)=.03, \mathrm{p}=.86]$.

\section{DISCUSSION}

Discrimination performance in Group W was superior to that in Group B, as expected. The results are consistent with the assumption that varying the irrelevant dimensions within settings during pretraining results in the extinction of irrelevant CPRs. Thus, the present results go a step further than earlier studies in showing that facilitation due to relevant verbal pretraining is greater if conditions are arranged so that irrelevant CPRs are likely to occur and be extinguished during pretraining. These findings also support and add generality to those from studies of discrimination shifts (Kemler \& Shepp, 1971; Shepp \& Gray, 1971). As noted earlier, an alternative interpretation of inferior performance in Group B is that these children had a stronger tendency to make irrelevant CPRs in the discrimination task because of the novel appearance of irrelevant values varying within settings.

A somewhat surprising result is the equivalent performance of Groups W and WO. Since the occurrence of relevant naming responses was required in Group WO, any failure to transfer relevant CPRs in Group W would be expected to produce poorer performance in that group. However, in view of the growing evidence of "production deficiency" in young children (e.g., Kendler, 1972), it seems unlikely that the equivalent performance of these groups was produced by complete transfer of relevant CPRs in Group W. On the contrary, it seems more likely that a certain proportion of children in Group WO were unable to profit from the overt-naming instructions. The fact that several children in Group WO had difficulty remembering to name the stimulus before each choice response and had to be reminded repeatedly supports this conclusion. For some young children, it may even be the case that overt labeling complicates the task to the extent that it produces interference, rather than facilitation.

Finally, it should be noted that, although the present results have been discussed primarily in terms of dimensional CPRs, the translation can readily be made to other types of
Table 1

Mean Proportion of Correct Responses in Discrimination Learning for Groups B, W, and WO

\begin{tabular}{ccccccc} 
& \multicolumn{5}{c}{ Eight-Trial Blocks } & All \\
\cline { 2 - 6 } Group & 1 & 2 & 3 & 4 & 5 & Trials \\
\hline B & .513 & .572 & .625 & .599 & .697 & .601 \\
W & .664 & .763 & .803 & .855 & .842 & .786 \\
WO & .671 & .703 & .862 & .862 & .888 & .797 \\
\hline
\end{tabular}

dimension-specific responses. For example, the inferiority of Group B could also be explained in terms of the extinction of irrelevant observing responses, attentional responses, or representational mediating responses. In other words, there is no intent to limit the hypothesized effects of the verbal pretraining to any specific type of dimensional mediation. Rather, the present results are viewed in a more general context as contributing to knowledge of the conditions of occurrence and transfer of dimension-specific responses, whatever their specific nature.

\section{REFERENCES}

Dickerson, D. J., Wagner, J. F., \& Campione, J. Discrimination shift performance of kindergarten children as a function of variation of the irrelevant shift dimension. Developmental Psychology, 1970, 3, 229-235.

Hardesty, C. S. The effects of verbal training on two discrimination tasks varying in difficulty. Unpublished master's thesis, University of Iowa, 1970.

Kemler, D. G., \& Shepp, B. E. Learning and transfer of dimensional relevance and irrelevance in children. Journal of Experimental Psychology, 1971, 90, 120-127.

Kendler, H. H., \& Kendler, T. S. Effects of verbalization on reversal shifts in children. Science, $1961,134,1619-1620$.

Kendler, H. H., \& Kendler, T. S. Mediation and conceptual behavior. In J. T. Spence (Ed.), The psychology of learning and motivation. Vol. 2. New York: Academic Press, 1968. Pp. 197-244.

Kendler, T. S. Verbalization and optional reversal shifts among kindergarten children. Journal of Verbal Learning and Verbal Behavior, 1964, 3, 428-436.

Kendler, T. S. An ontogeny of mediational deficiency. Child Developm ent, 1972, 43, 1-17.

Kendler, T. S. The effect of training and stimulus variables on the reversal-shift ontogeny. Journal of Experimental Child Psychology, 1974, 17, 87-106.

Miller, A. A. Children's simultaneous discrimination learning as a function of pretraining condition. Unpublished master's thesis, University of Iowa, 1971.

Shepp, B. E., \& Gray, V. A. Some effects of variable-within and variable-between irrelevant stimuli on dimensional learning and transfer. Journal of Experimental Psychology, 1971, 89, 32-39.

Shepp, B. E., \& Turrisi, F. D. Learning and transfer of mediating responses in discrimination learning. In N. R. Ellis (Ed.), International review of research in mental retardation. Vol. 2, New York: Academic Press, 1966, Pp. 86-120.

Spiker, C. C. Application of Hull-Spence theory to the discrimination learning of children. In $H$. W. Reese (Ed.), Advances in child development and behavior. Vol. 6, New York: Academic Press, 1971, Pp. 99-152.

Spiker, C. C., \& Cantor, J. H. Applications of Hull-Spence theory to the transfer of discrimination learning in children. In $\mathrm{H}$. W. Reese (Ed.), Advances in child development and behavior. Vol. 8. New York: Academic Press, 1973, Pp. 223-288.

Tighe, L. S. \& Tighe, T. J. Discrimination learning: Two views in historical perspective. Psychological Bulletin, 1966, 66, 353-370.

Tighe, T. J., \& Tighe L. S. Optional shift behavior of children as a function of age, type of pretraining, and stimulus salience. Journal of Experimental Child Psychology, 1970, 9, 272-285.

Zeaman, D., \& House, B. J. The role of attention in retardate discrimination learning. In N. R. Ellis, (Ed.), Handbook of mental deficiency. New York: McGraw-Hill, 1963, Pp. 159-223.

(Received for publication October 1, 1974.) 